\title{
Miksopapiller ependimomda cerrahi sonuçlar: kapsül bütünlüğünün önemi
}

\author{
Surgical results in the myxopapillary ependymoma: the importance of capsule integrity \\ İsmail İştemen, Ali Arslan, Semih Kıvanç Olguner, Yurdal Gezercan, Ali İhsan Ökten, Kemal Alper \\ Afşer, Emre Bilgin
}

Gönderilme tarihi:20.04.2019

Kabul tarihi:02.10.2019

\section{Özet}

Giriş: Primer intramedüller tümörler nadir görülen, yetişkinlerde ağırlıklı olarak astrositomlar ve ependimomlardan oluşan tümörlerdir. Ependimomlar servikal ve servikotorasik bölgede sık görülürken, miksopapiller ependimomlar hemen her zaman konus medüllaris, kauda ekuina ve filum terminale düzeyinde ortaya çıkarlar. Miksopapiller ependimomlar mitotik aktivitesi düşük olan düşük evreli (evre 1) tümörlerdir ancak rekürrens sık bildirilmiştir. Tedavisi total cerrahi rezeksiyondur ve bu tekrarlama ihtimalini düşürür. Fakat, kapsülü ince ve zayıf olan vakalarda total rezeksiyon mümkün değildir, bu nedenle rekürrens, seeding metastaz ve uzak metastaz riski artmaktadır. Total çıkarılamayan olgularda radyoterapi eklenmesi önerilir. Bu çalışmanın amacı kliniğimizde miksopapiller ependimomlar nedeni ile opere edilen hastaların sonuçlarını araşıımak ve klinik tecrübelerimizi paylaşmaktır.

Gereç ve yöntem: Bu çalışmada, 2014-2016 yılları arasında, Adana Şehir Eğitim ve Araştırma Hastanesi Beyin Cerrahi Bölümü'nde bütün olarak çıkartılan ve miksopapiller ependimom tanısı alan 9 olgu retrospektif olarak incelenmiştir. Hastalar, operasyon öncesi ve sonrası klinik ve radyolojik bulguları ile değerlendirilmiştir. Hastalar total laminektomi ile total kitle çıkarılarak tedavi edilmişlerdir.

Bulgular: 7 kadın 2 erkek hasta rutin takibe alınmıştır. Yaş aralığı 9-57 olup ortalama yaş $33^{\prime}$ tü. 3 hastada kitle L2 seviyesinde, 2 hastada L3 seviyesinde, 1 hastada L4 seviyesinde ve 3 hastada L4-L5 vertebralar boyunca yerleşmekteydi. Tüm hastaların bel ağrısı şikayeti vardı, ek olarak 4 hastada paraparezi ve bir hastada da gaita idrar inkontinansı mevcuttu. Operasyon sonrasında ağrı şikayeti tüm hastalarda geçti. 3 hastada operasyon sonrası erken dönemde kas gücünde belirgin düzelme saptandı. 1 hastada ise operasyondan 2 ay sonra nörolojik iyileşme olduğu görüldü. İnkontinans gelişmiş hastada ise düzelme olmadı. Hastanede kalış süresi ortalama 5,1 gündü. Hastaların radyolojik takiplerinde operasyon sonrası 2 yıl içinde nüks saptanmadı.

Sonuç: Kapsül bütünlüğü bozulmaksızın total olarak kitle çıkarılması rekürensi ve seeding metastazları önlemede önemlidir. Bu hastalar radyoterapi verilmeksizin takip edilebilirler. Ancak uzun dönem takipler ve daha fazla sayıda hastanın olduğu gruplarla yapılan çalışmalar gereklidir.

Anahtar kelimeler: İntramedüller tümör, miksopapiller ependimom, total cerrahi çıkarım.

İştemen I, Arslan A, Kıvanç Olguner S, Gezercan Y, Ökten Al, Afşer KA, Bilgin E. Miksopapiller ependimomda cerrahi sonuçlar: kapsül bütünlüğünün önemi. Pam Tıp Derg 2020;13:27-31.

\begin{abstract}
Purpose: Primary intramedullary tumors are rare tumors and mainly astrocytomas and ependymomas in adults. While ependymomas are common in the cervical and cervicothoracic region, myxopapillary ependymomas often occur at the level of conus medullaris, cauda ekuina and filum terminale. Myxopapillary ependymomas are low grade (grade 1) tumors with low mitotic activity but recurrence has been reported frequently. The treatment is total surgical resection and this reduces the recurrence. But in cases with thin and weak capsule, total resection is not possible, therefore the risk of recurrence, seeding metastasis and distant metastasis increase. It is recommended to add radiotherapy for cases which cannot be totally removed. The aim of this study is to asses the clinical outcomes of the patients who underwent surgery with myxopapillary ependymoma and to share our clinical experience.
\end{abstract}

İsmail İştemen, Uzm. Dr. Adana Sağlık Bilimleri Üniversitesi, Adana Şehir Eğitim ve Araştırma Hastanesi Beyin ve Sinir Cerrahisi, ADANA, e-posta: drismailistemen@gmail.com (orcid.org/0000-0002-2341-4818) (Sorumlu yazar)

Ali Arslan, Uzm. Dr. Adana Sağlık Bilimleri Üniversitesi, Adana Şehir Eğitim ve Araştırma Hastanesi Beyin ve Sinir Cerrahisi, ADANA, e-posta: aliarslan26062006@hotmail.com (orcid.org/0000-0002-7457-5283)

Semih Kıvanç Olguner, Uzm. Dr. Adana Sağlık Bilimleri Üniversitesi, Adana Şehir Eğitim ve Araştırma Hastanesi Beyin ve Sinir Cerrahisi, ADANA, e-posta: kivanc3olguner@hotmail.com (orcid.org/0000-0002-5314-4636)

Yurdal Gezercan, Uzm. Dr. Adana Sağlık Bilimleri Üniversitesi, Adana Şehir Eğitim ve Araştırma Hastanesi Beyin ve Sinir Cerrahisi, ADANA, e-posta: gezercan@hotmail.com (orcid.org/0000-0002-4124-2036)

Ali İhsan Ökten, Doç. Dr Adana Sağlık Bilimleri Üniversitesi, Adana Şehir Eğitim ve Araştırma Hastanesi Beyin ve Sinir Cerrahisi, ADANA, e-posta: aihsano1@gmail.com (orcid.org/0000-0003-0292-201X)

Kemal Alper Afşer, Asst. Dr Adana Sağlık Bilimleri Üniversitesi, Adana Şehir Eğitim ve Araștırma Hastanesi Beyin ve Sinir Cerrahisi, ADANA, e-posta: kemalalperafser@gmail.com (orcid.org/0000-0002-4724-0840)

Emre Bilgin, Uzm. Dr Adana Sağlık Bilimleri Üniversitesi, Adana Şehir Eğitim ve Araştırma Hastanesi Beyin ve Sinir Cerrahisi, ADANA, e-posta: dremreblgn@gmail.com (orcid.org/0000-0002-2394-1503) 
Material and methods: In the present study, 9 cases with the diagnosis of myxopapillary ependymoma, underwent total excision between 2014-2016 in Adana City Treaning and Research Hospital, Department of Neurosurgery were evaluated retrospectively. The patients were evaluated with radiological images and clinical parameters preoperatively and postoperatively. Patients were treated with total laminectomy and total tumour excision.

Results: 7 male and 2 female patients were taken to routine follow-up. The age range is 9-57 and the average age is 33. The mass was located at L2 level in 3 patients, at L3 in 2 patients, at L4 in 1 pateient and along the L4 and L5 vertebrae in 3 patients. All patients had back pain, additionally 4 had paraparesia and 1 had urineanal incontinence. Following surgery all patiens recovered from back pain. Significant improvement in muscle strength was detected in 3 patients at the early postoperative period. In 1 patient, neurological healing was observed in the 2 months after operation. No improvement was observed in sphincter function. The mean length of stay in hospital was 5.1 days. No recurrence was detected in the postoperative 2 years radiologically.

Conclusion: Total removal of mass without deterioration of capsule integrity is imperative in preventing recurrence and seeding metastases. These patients can be followed without radiotherapy. However, long-term follow-up and studies with more patient groups are required.

Key words: Intramedullary tumor, myxopapillary ependymoma, total surgical removal.

İştemen İ, Arslan A, Kıvanç Olguner S, Gezercan Y, Ökten Aİ, Afşer KA, Bilgin E. Surgical results of myxopapillary ependymoma: the importance of capsule integrity. Pam Med J 2020;13:27-31.

\section{Giriş}

Primer intramedüller tümörler nadir görülen tümörlerdir. Görülme sıklı̆̆ı 100,000 de 0,30,5 arasındadır [1]. İntramedüller tümörler yetişkinlerde ağırlıklı olarak astrositomlar ve ependimomlardan oluşur. Büyük çoğunluğu da ependimomdur [2-4]. Ependimomların alt tipi olan miksopapiller ependimom tüm spinal ependimomların \%27-30'unu oluşturur [5-7]. Ependimomlarservikal ve servikotorasikbölgede sık görülürken miksopapiller ependimomlar hemen her zaman konus medüllaris, kauda ekuina ve filum terminale düzeyinde ortaya çıkarlar [3, 8-13]. Miksopapiller ependimomların insidansı yıllık 100,000 de 0,05-0,08 oranındadır [14]. Dünya Sağlık Örgütü (DSÖ) sınıflamasına göre miksopapiller ependimomlar evre 1 olarak sınıflandırılırlar ve yavaş büyüyen mitotik aktivitesi düşük olan tümörlerdir. Buna karşılık hastaların yaklaşık yarısında (\%48) rekürrens gözlenmektedir [15]. Mikroskobik olarak papiller düzende müsinöz salgı salgılayan küboidal hücrelerle karakterizedir. Spinal miksopapiller ependimomlar ortalama 35 yaş erkek hastalarda sıktır [3]. Genellikle yavaş büyüme eğilimindedir ve nörolojik defisit gelişmez, bu sebeple geç tanı alırlar. Hastalar tanı aldığında en sık şikayet (\%67) ağrıdır. Daha nadir olarak (\%52) duyu defisiti ve (\%46) motor defisitler olabilir $[3,10,16]$. Gecikmiş tanı, tedavi sonuçlarını da olumsuz etkilemektedir. Tedavisi total cerrahi rezeksiyondur. Olguların yaklaşık \%90'ında total rezeksiyon yapılabilir, total çıkarılan tümörlerde tekrarlama intimali (\%15) oldukça düşüktür $[7$, 17]. Tekrarlama riski doğrudan tümörün çıkarılan miktarı ile alakalıdır. Kapsülasyonu zayıf olan vakalarda total rezeksiyon mümkün olamamakla beraber seeding metastaz ve uzak metastaz riski de artmaktadır [7]. Total çıkarılamayan olgularda radyoterapi (RT) eklenmesi önerilir [18, 19].

$\mathrm{Bu}$ çalışmada kliniğimizde 2014-2016 yılları arasında opere edilen ve patoloji sonucu miksopapiller ependimom olarak gelen 9 olgu retrospektif olarak incelenerek klinik sonuçları literatür verileri ile birlikte değerlendirilmiştir.

\section{Gereç ve yöntem}

Kliniğimizde 2014-2016 yılları arasında spinal kitle nedeni ile opere edilen ve patoloji sonucu miksopapiller ependimom olarak bildirilen olgular retrospektif olarak incelenmiştir. Takipleri yapılabilmiş 9 hasta çalışmaya alınmıştır. Hastaların operasyon sonrası en kısa altı ay en uzun iki yıllık takibi yapılmıştır. Hastalar, operasyon öncesi ve sonrası klinik ve radyolojik bulguları ile şikayet başlangıç süreleri, hastanede yatış süreleri ile irdelenmiştir. Radyolojik tanı yöntemi olarak manyetik rezonans görüntüleme (MRG) kullanılmıştır. Cerrahi yöntem olarak lezyon seviyesinde total laminektomi, total kitle eksizyonu ve duraplasti uygulanmıştır. Hastaların tamamında kitle lezyonu total olarak çıkarıldığı için ek RT ve kemoterapi (KT) uygulanmamıştır. Nörodefisiti olan hastalara fizyoterapi erken dönemde başlatılmıştır. Hastaların operasyon sonrası ikinci, altıncı aylarda ve birinci yıl sonrasında rutin kontrolleri yapılmıştır. Kontrollerinde muayene 
bulguları operasyon öncesi ile karşılaştırılmıştır. Radyolojik olarak MRG ile nüks ve rezidü kitle olup olmadığı değerlendirilmiştir.

\section{Bulgular}

Kliniğimizde opere edilen ve sonrasında takibi yapılabilen 9 miksopapiller ependimom olgusu incelenmiştir. 7 kadın 2 erkek hasta takibe alınmıştır. Yaş aralığı 9-57 olup ortalama yaş 33'tü. 3 hastada kitle 2. Lomber vertebra (L2) seviyesinde, 2 hastada L3 seviyesinde, 1 hastada L4 seviyesinde ve 3 hastada L4-L5 vertebralar boyunca yerleşmekteydi. Literatürde de belirtildiği gibi MRG bulgularında kitle konus ve filum düzeyinde yerleşmekteydi (Resim 1).
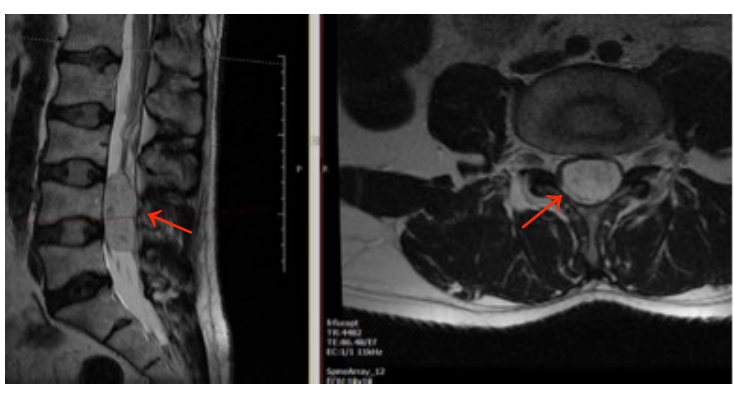

Resim 1. MRG T2 W Sagital ve aksiyel kesitler.

Tüm hastaların ortak şikayeti bel ağrısı olup; dört hastada paraparezi ve bir hastada da gaita idrar inkontinansı ilave şikayetlerdendi. Şikayetlerin ve semptomların süresi minimum 1 gün maksimum 3 ay olarak saptandı (Tablo1).

Operasyon sonrasında ağrı şikayeti tüm hastalarda geçti. Paraparezisi olan 4 hastadan 3'ünde erken dönemde önemli oranda düzelme saptandı. 1 hastada ise 2 . ay kontrolünde düzelme olduğu görüldü. İnkontinans gelişmiş hastada ise düzelme olmadı. Hastanede yatış süreleri 1 hastada 7 gün, 1 hastada 6 gün, 5 hastada 5 gün, 2 hastada 4 gündü. Nörodefisit olmayan bir hastanın operasyonunda nöromonitörizasyon kullanıldı. Bu hastada operasyon sonrasındadanörodefisitolmadı.Tüm hastaların operasyonunda duraplasti sonrası doku yapıştırıcı kullanıldı. Operasyon sonrası dönemde 1 hasta hariç diğer hastalarımızın operasyon lojundan beyin omurilik sıvısı (BOS) kaçağı olmadı. BOS kaçağı olan hastada tekrar operasyona gerek kalmadan spontan iyileşme oldu. Servis yatış süresi 7 günle en uzun süren hastamız BOS kaçağı olan bu hastamız oldu. Hiçbir hastada posterior enstrümantasyona ihtiyaç duyulmadı ve kullanılmadı. Hastalara ek RT ve KT verilmedi. Hastaların radyolojik takiplerinde hiçbir hastada nüks saptanmadı (Resim 2).

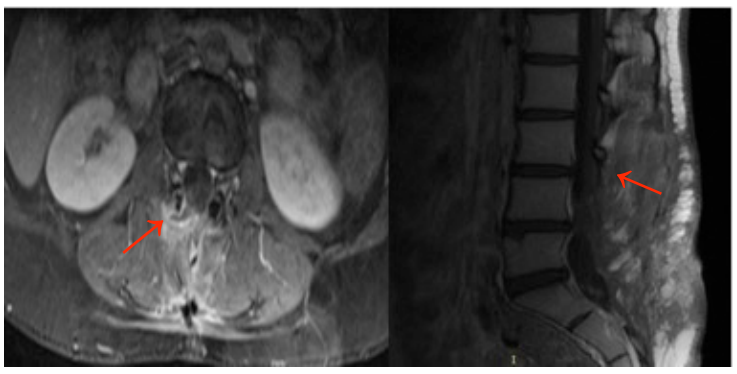

Resim 2. Kontrol kontrastlı aksiyel ve sagital MRG kesiti.

\section{Tartışma}

Spinal ependimal tümörler ependim hücrelerden köken alan glial tümörlerdir ve primer spinal kord tümörlerinin \%40-60'ını oluşturur $[20,21]$. Hemen her zaman konus medüllaris, kauda ekuina ve filum terminale düzeyinde ortaya çıkarlar [2]. Bizim hastalarımızın tamamında da yerleşim yeri literatürle uyumlu olarak lomber bölgede ve konus medüllaris, kauda ekuina veya filum terminale ile bağlantılı haldeydi. 30-40'lı yaşlarda daha sık görülür ve erkeklerde kadınlardan daha sık görülür $[22,23]$. Bizim hastalarımızın yaş ortalaması 33 ve en düşük 9 yaş, en yüksek 57 yaş aralığındaydı. Ancak hastalarımızın 7'si kadın 2'si erkek hastaydı. Genellikle yavaş büyüme eğilimindedir ve nörolojik defisit gelişmez, bu sebeple geç tanı alırlar. Hastalar tanı aldığında en sık şikayet (\%67) ağrıdır. Bununla beraber motor duyu defisitleri ve idrar gayta inkontinansı olabilir. Hastaların semptomlarının başlangıç süreleri 13 aydan 8,3 yıla kadar değişen aralıkta bildirilmiştir [4, 23, 24]. Bizim hastalarımızda ise bu süre 1 gün ile 3 ay arasında değişmekteydi. Ülkemizde zaman zaman eleştirdiğimiz çok sık MRG çekilmesinin belki de olumlu bir yönü olarak daha erken tanı konulduğu söylenebilir. Tanıda en iyi yöntem MRG'dir. Tipik olarak intradural yerleşmiş, T1 imajlarda kordla izointens veya hipointens, T2 imajlarda hemoraji ve kalsifikasyona bağlı heterojen hiperintensite ve homojen kontrastlanan kitle lezyonu şeklinde görülür [2, 12, 22, 25-27]. Tedavide ilk seçenek total cerrahi rezeksiyondur. Hala progresyondaki en önemli faktör total cerrahi rezeksiyondur [4, 7, 20, 22, 28]. Rezeksiyon sırasında kapsülü rüptüre etmek BOS ekilimine yol açabileceğinden tek parça halinde 
Tablo 1. Hastalara ait demografik ve klinik veriler.

\begin{tabular}{|c|c|}
\hline Olgu & Yaş/ cinsiyet/ şikayet/ süresi/ lezyon seviyesi/ muayene/ yatış süresi/ kontrol \\
\hline Olgu1 & $\begin{array}{l}\text { 23/ K/ bel ve bacaklarda ağrı uyuşma/ } 1 \text { hafta/ L2 seviyesinde/ bacaklarda 3/5 kuvvet kaybı/ } \\
4 \text { gün yatış/ } 1 \text { yıl sonra kontrolde intakt, nüks saptanmadı }\end{array}$ \\
\hline Olgu2 & $\begin{array}{l}43 / \mathrm{K} / \text { bel ağrısı, bacaklarda güçsüzlük/ 2ay/ L2 seviyesinde / bacaklarda } 1 / 5 \text { kuvvet kaybı/ } \\
7 \text { gün yatış/ } 2 \text { yıl sonra kontrolde intakt, nüks saptanmadı }\end{array}$ \\
\hline Olgu3 & $\begin{array}{l}\text { 31/ K/ bel ağrısı, bacaklarda güçsüzlük/ 1ay/ L4-5 korpus boyunca/ bacaklarda 1/5 kuvvet } \\
\text { kaybı/ } 4 \text { gün yatış/ } 6 \text { ay sonra kontrolde intakt, nüks saptanmadı }\end{array}$ \\
\hline Olgu4 & $\begin{array}{l}\text { 30/ K/ bel ağrısı/ 3ay/ L4-5 korpus boyunca/ nörodefisit yok/ } 5 \text { gün yatış/ } 6 \text { ay sonra } \\
\text { kontrolde intakt, nüks saptanmadı }\end{array}$ \\
\hline Olgu5 & $\begin{array}{l}\text { 40/ E/ bel ağrısı/ } 2 \text { ay/ L4 seviyesinde/ nörodefisit yok / } 5 \text { gün yatış/ } 6 \text { ay sonra kontrolde } \\
\text { intakt, nüks saptanmadı }\end{array}$ \\
\hline Olgu6 & $\begin{array}{l}57 / \mathrm{K} / \text { bel ağrısı, bacaklarda güçsüzlük/2gün/ L2 seviyesinde/ bacaklarda 3/5 kuvvet kaybı/ } \\
6 \text { gün yatış/ 1,5 yıl sonra kontrolde intakt, nüks saptanmadı }\end{array}$ \\
\hline Olgu7 & $\begin{array}{l}\text { 23/ K/ bel ağrısı/ 3ay/ L4-5 korpus boyunca/ nörodefisit yok/ } 5 \text { gün yatış/ } 1 \text { yıl sonra } \\
\text { kontrolde intakt, nüks saptanmadı }\end{array}$ \\
\hline Olgu8 & $\begin{array}{l}41 / \text { E/ bel ağrısı, idrar kaçırma/ 1gün/ L3 seviyesinde/ idrar inkontinansı mevcut/ } 5 \text { gün } \\
\text { yatış/ } 6 \text { ay sonra kontrolde inkontinans mevcut, nüks saptanmadı }\end{array}$ \\
\hline Olgu9 & $\begin{array}{l}\text { 9/ K/ bel ağrısı/ 2ay/ L3 seviyesinde/ nörodefisit yok/ } 5 \text { gün yatış/ } 1 \text { yıl sonra kontrolde } \\
\text { intakt, nüks saptanmadı }\end{array}$ \\
\hline
\end{tabular}

çıkarılması önerilir. Tek parça halinde ya da total çıkarılamadığı durumda RT eklenmelidir [12, 18, 23]. Son dönem çalışmalarda RT'nin yaşam beklentisini arttırdığı, lokal tümör kontrolünü \%30'dan \%100'e çıkardığı ve genç yaş nedeni ile RT verilmeyen hastalarda daha sık nüks saptandığı bildirilmektedir [28-30]. Bu konuda Avrupa Nöroonkoloji Topluluğunun (EANO) hazırladığı rehberde tedavinin ana amacının tümörün tamamen çıkarılması olduğu bildirilmiştir. Aynı rehberde Grade 3 olan kitlelerde mutlaka RT verilmesi, Grade 2 olan ve tamamen çıkarılamayan kitlelerde RT verilmesi, Grade 2 olan ve tamamen çıkarılan kitlelerde bekle ve gör stratejisi önerilmektedir. Rehberde Grade 1 olan miksopapiller ependimomlar için ise RT eklenmesinin olumlu sonuçları olduğunu bildiren çalışmalar olsa da prospektif çalışmalara intiyaç olduğu bildirilmiştir [19]. Kliniğimizde opere ettiğimiz hastalarda kapsülü korunarak total olarak kitle çıkarılması uygulanmıştır. Literatürde bildirilen olgulara göre erken dönemde tanı alan hastalarımızda kitlenin total olarak çıkarılması ve metastaz şüphesi olmadığından RT uygulanmamıştır. Erken cerrahi ve total eksizyon yapılan ve RT almayan hastalarımızın 6 ay ile 2 yıl arasında yapılan takiplerinde nüks henüz saptanmamıştır.
Nadir görülen bir kitle olması sebebi ile az sayıda hasta çalışmaya alınabilmiştir ve henüz yeterince uzun süre takiplere erişilmemiştir. Ancak yine de erken dönemde tanı konulan, total olarak çıkarılabilen ve cerrahi sırasında kapsülü zedelenmeyen olgularda literatürde bildirilenin aksine RT verilmeyebileceği kanaati oluşmaktadır.

Çıkar ilişkisi: Yazarlar çıkar ilişkisi olmadığını beyan ederler.

\section{Kaynaklar}

1. Helseth A, Mork SJ. Primary intraspinal neoplasms in Norway, 1955 to1986. A population-based survey of 467 patients. J Neurosurg 1989;71:842-845. https://doi. org/10.3171/jns.1989.71.6.0842

2. Choi JY, Chang $\mathrm{KH}, \mathrm{Yu} \mathrm{IK}$, et al. Intracranial and spinal ependymomas: review of MR images in 61 patients. Korean J Radiol 2002;3:219-228. https://doi. org/10.3348/kjr.2002.3.4.219

3. Ferrante L, Mastronardi L, Celli P, Lunardi P, Acqui M, Fortuna A. Intramedullary spinal cord ependymomas: a study of 45 cases with longterm follow-up. Acta Neurochir 1992;119:74-79.

4. Sakai $\mathrm{Y}$, Matsuyama $\mathrm{Y}$, Katayama $\mathrm{Y}$, et al. Spinal myxopapillary ependymoma: neurological deterioration in patients treated with surgery. Spine 2009;34:16191624. 
5. Barone BM, Elridge AR. Ependymoma. A clinical survey. J Neurosurgery 1970;33:428-438. https://doi. org/10.3171/jns.1970.33.4.0428

6. Johnson DL, Schwarz S. Intracranial metastases from malignant spinal cord astrocytoma. J Neurosurgery 1987;66:621-625. https://doi.org/10.3171/ jns.1987.66.4.0621

7. Sonneland PR, Scheithauer BW, Onofrio BM. Myxopapiller ependymoma, a clinicopathologic and immunocythochemical study of 77 cases. Cancer 1985;56:883-893.

8. Bagley CA, Kothbauer KF, Wilson S, Bookland MJ, Epstein FJ, Jallo GI. Resection of myxopapillary ependymomas in children. J Neurosurg 2007;106:261267. https://doi.org/10.3171/ ped.2007.106.4.261

9. Celli P, Cervoni L, Morselli E, Ferrante L. Spinal ependymomas and papilledema: report of 4 cases and review of literature. J Neurosurg Sci 1993;37:97-102.

10. Hoshimaru M, Koyama T, Hashimoto N, Kikuchi $\mathrm{H}$. Results of microsurgical treatment for intramedullary spinal cord ependymomas: analysis of 36 cases. Neurosurg 1999;44:264-269. https://doi. org/10.1097/00006123-199902000-00012

11. Koeller KK, Rosenblum RS, Morrison AL. Neoplasms of the spinal cord and filum terminale: radiologic-pathologic correlation. Radiographics 2000;20:1721-1749. https:// doi.org/10.1148/radiographics.20.6.g00nv151721

12. Nakamura $M$, Ishii $K$, Watanabe $K$, et al. Longterm surgical outcomes for myxopapillary ependymomas of the cauda equina. Spine (Phila Pa 1976) 2009;34:756760. https://doi.org/10.1097/ BRS.0b013e3181b34d16

13. Liu T, Yang C, Deng $X$, et al. Clinical characteristics and surgical outcomes of spinal myxopapillary ependymomas. Neurosurg Rev 2019 https://doi. org/10.1007/s10143-019-01150-z

14. McLendon $R$, Rosenblum $M$, Schiffer $D$, et al. Myxopapillary ependymoma. In: Louis $D$, Ohgaki $H$, Wiestler O, Cavenee W, eds. WHO Classification. Lyon: WHO 2007;72-73.

15. Keleş E, Aydın S, Mandel NM, Selek U. Düşük dereceli glial tümörler. Temel Nöroşirürji Cilt 1, Türk Nöroşirürji Derneği Yayınları No:10 Ankara 2010;1019-1037.

16. Kucia EJ, Bambakidis NC, Chang SW, Spetzler RF. Surgical technique and outcomes in the treatment of spinal cord ependymomas, part 1: intramedullary ependymomas. Neurosurg 2011;68:57-63. https://doi. org/10.1227/NEU.0b013e318208f181

17. Nakamura M, Ishii K, Watanabe K. et al. Surgical treatment of intramedullary spinal cord tumors:pPrognosis and complications. Spinal Cord 2008;46:282-286. https://doi.org/10.1038/ sj.sc. 3102130
18. Sudhan MD, Satyarthee GD, Joseph L, Sharma MC, Kakkar A, Sharma BS. Management and outcome analysis of conus and filum ependymoma: a tertiary center study. Asian J Neurosurg 2019;14:821-827. https://doi.org/10.4103/ajns.AJNS_326_16

19. Ruda R, Reifenberger G, Frappaz D, et al. EANO guidelines for the diagnosis and treatment of ependymal tumors. Neuro Oncol 2018;20:445-456. https://doi.org/10.1093/neuonc/nox166

20. Bavbek M, Altinors MN, Caner HH, Bilezikci B, Agildere M. Lumbar myxopapillary ependymoma mimicking neurofibroma. Spinal Cord 2001;39:449-452. https:// doi.org/10.1038/sj.sc.3101173

21. Wippold FJ, Smirniotopoulos JG, Moran CJ, Suojanen $\mathrm{JN}$, Vollmer DG. MR imaging of myxopapillary ependymoma: findings and value to determine extent of tumour and its relation to intraspinal structures. AJR Am J Roentgenol 1995;165:1263-1267. https://doi. org/10.2214/ajr.165.5.7572515

22. Sun B, Wang C, Wang J, Liu A. MRI features of intramedullary spinal cord ependymomas. J Neuroimaging 2003;13:346-351.

23. Volpp PB, Han K, Kagan AR, Tome M. Outcomes in treatment for intradural spinal cord ependymomas. Int J Radiat Oncol Biol Phys 2007;69:1199-1204. https:// doi.org/10.1016/j.ijrobp.2007.04.058

24. Asazuma T, Toyama Y, Suzuki N, Fujimura Y, Hirabayshi $K$. Ependymomas of the spinal cord and cauda equina: an analysis of 26 cases and a review of the literature. Spinal Cord 1999;37:753-759.

25. Bagley CA, Wilson S, Kothbauer KF, Bookland MJ, Epstein F, Jallo Gl. Long term outcomes following surgical resection of myxopapillary ependymomas. Neurosurg Rev 2009;32:321-334. https://doi. org/10.1007/s10143-009-0190-8

26. Bandopadhayay $\mathrm{P}$, Silvera VM, Ciarlini PD, et al. Myxopapillary ependymomas in children: imaging, treatment and outcomes. J Neurooncol 2016;126:165174. https://doi.org/10.1007/s11060-015-1955-2

27. KlekampJ.Spinal ependymomas. Part2: ependymomas of the filum terminale. Neurosurg Focus 2015;39:7. https://doi.org/10.3171/2015.5.FOCUS15151

28. Weber DC, Wang Y, Miller R, et al. Long-term outcome of patients with spinal myxopapillary ependymoma: treatment results from the MD Anderson Cancer Center and institutions from the Rare Cancer Network. Neuro Oncol 2015;17:588-595. https://doi.org/10.1093/ neuonc/nou293

29. Agbahiwe HC, Wharam M, Batra S, Cohen K, Terezakis SA, Management of pediatric myxopapillary ependymoma: the role of adjuvant radiation. Int $\mathrm{J}$ Radiat Oncol Biol Phys 2013;85:421-427. https://doi. org/10.1016/j.jijrobp.2012.05.001

30. Taylor RE. Review of radiotherapy dose and volume for intracranial ependymoma. Pediatr Blood Cancer 2004;42:457-460. https://doi.org/10.1002/pbc.10470 